\title{
Relationship of Timings and Outcome of Tracheostomy Among Patients Requiring Prolonged Mechanical Ventilation
}

Muhammad Asim Shafique, Muhammad Amir Nadeem, Muhammad Afzal

\section{- - - - - - - - - - - - - - - - - - - - - - - - - - - - - - -} ABSTRACT

Objective: To assess the indications of tracheostomy among patients requiring prolonged mechanical ventilation and to observe the relationship of the timings and outcome of tracheostomy with age and gender.

Study Design and Setting: It was a descriptive study conducted at intensive care unit (ICU) of Department of Otorhinolaryngology at Bahawal Victoria Hospital from January 2017 to December 2018.

Methodology: Secondary data was collected with the help of charts of ICU patients in which elective tracheostomy was done to replace orotracheal intubation for mechanical ventilation. Inclusion and exclusion criteria were designed. Clinical record was reviewed for the assessment of indications of the procedure (medical or surgical) along with age and gender distribution. The timing of tracheostomy in these patients with its outcome in terms of decannulation and weaning were recorded. Proforma was used to enter the findings. Finally results were obtained and assessed on SPSS Version 23.

Results: Out of total 551 tracheostomies $42(7.6 \%)$ were indicated for the patients of ICU requiring prolonged mechanical ventilation. From the 42 mechanical ventilated patients majority had Guillain-Barre syndrome (GBS) 20(47.6\%). Twenty six patients were adults $(61.9 \%)$ and sixteen were children (38\%). Twenty four were male patients $(57.1 \%)$ and eighteen were females (42.8\%). The timing of tracheostomy among majority of the patients (40) was from 7-10 days, with mean of 9th day with good outcome. Only two patients who underwent tracheostomy after two weeks had to face poor outcome (failed decannulation, late weaning) (4.7\%).

Conclusion: Neuroparalytic lesions were the common indication among the patients requiring prolonged mechanical ventilation with tracheostomy. Tracheostomy if performed earlier in such patients carries good outcome.

Key Words: Decannulation, Intensive care unit, Mechanical ventilation, Tracheostomy, Weaning

\section{INTRODUCTION:}

Tracheostomy is one of the most commonly performed surgical procedures in the critically ill patients ${ }^{1}$. It is also one of the oldest operations as Asciepiades of Persia used to perform tracheostomies some 4000 years ago ${ }^{1,2}$. The initial most reference of this procedure can be located in the RigVeda written in $1500 \mathrm{BC}^{2}$. The procedure was considered dangerous and carried hazards till the initial part of $19^{\text {th }}$ century therefore rarely performed ${ }^{3}$. It was Chevalier Jackson who first mentioned the modern classic surgical technique for tracheostomy in $1909^{2}$. Tracheostomy has been believed to be a safe, effective and single life saving procedure during the past three decades ${ }^{3}$. The indications of tracheostomy can

Muhammad Asim Shafique

Assistant Professor, ENT and Head \& Neck Surgery,

QAMC / Bahawal Victoria Hospital,

I Bahawalpur

E.mail: drasimkemc@yahoo.com

I Muhammad Amir Nadeem

Associate Professor, ENT and Head \& Neck Surgery

I QAMC / Bahawal Victoria Hospital

I Bahawalpur

I Muhammad Afzal

I Consultant ENT Surgeon,

Shahida Islam Medical and Dental College Lodhran, I Bahawalpur

Received: 01-06-2019

I Received: 01-06-2019

I ------------------1 be emergency or elective ${ }^{3}$. Being an elective procedure tracheostomy is commonly performed in the patients of intensive care unit (ICU), requiring prolonged mechanical ventilation ${ }^{4}$. Almost $10 \%$ of the patients in ICU having mechanical ventilation require tracheostomy $y^{5,4}$. The advantages of tracheostomy over orotracheal intubation in the patients with mechanical ventilation may be the reduction of anatomical dead space, low incidence of oral and laryngeal ulceration, more patient comfort and ability to communicate ${ }^{6,7}$. There are many surgical and medical causes for which patients require prolonged assisted ventilation with tracheostomy. Poliomyelitis, respiratory muscle paralysis, Guillain Barre syndrome (GBS), coma, neuromuscular diseases, sepsis and chronic obstructive pulmonary disease (COPD) can be the common medical causes ${ }^{3,4}$. Surgical causes may be some postoperative surgical complication or any non surgical trauma ${ }^{4,6}$. Head injury or Traumatic brain injury (TBI) is the usual cause in non-surgical category where patients require prolonged mechanical ventilation with tracheostomy ${ }^{5}$.

The timing of tracheostomy in all these patients with prolonged mechanical ventilation has been a matter of controversy thus remains unclear ${ }^{8}$. However early tracheostomy results successful decannulation and weaning from ventilator and vice verca ${ }^{9}$. Moreover the selection of patients is also important as those with head trauma or traumatic brain injury, tracheostomy as early as possible 
after the start of mechanical ventilation is advocated ${ }^{5,9}$. Worldwide; various different studies have been carried out on this subject but focusing mainly on the timing of elective tracheostomy and their short term outcomes in the mechanically ventilated patients ${ }^{8,2,6}$. Some of studies are done exclusively in mechanically ventilated children and infants ${ }^{9,10}$. In Pakistan the first study has been reported and carried out by Prem Kumar et al assessing the outcomes and complications of elective tracheostomy in children who were mechanically ventilated ${ }^{11}$. Therefore; the rationale of our study was to assess the indications for tracheostomy, effects of its timing among all age group and gender requiring prolonged mechanical ventilation in our settings and to assess the medical or surgical indications among the patients requiring prolonged mechanical ventilation with tracheostomy, effects of its timing among all age group and gender in our settings.

\section{METHODOLOGY:}

It was a descriptive study conducted at intensive care unit (ICU) of Department of Otorhinolaryngology at Bahawal Victoria Hospital from January 2017 to December 2018.Secondary data was collected with the help of charts of ICU patients in which elective tracheostomy was done to replace orotracheal intubation for mechanical ventilation. After the formal permission from the hospital ethical committee; the records were reviewed for all the patients irrespective of age and sex group from the main intensive care unit of Bahawal Victoria Hospital. Patients below 15 years of age were taken as children.

Operational Definitions: Tracheostomy: Surgical procedure in which anterior tracheal wall is opened at the level of second third and fourth ring and connecting the opening with the skin by Tracheostomy tube.

Prolonged mechanical ventilation: It is the assisted positive airway pressure ventilation given to the patient with the help of ventilator for more than a week.

Decannulation: It is the reversal of tracheostomy intubation when the patient is free of initial causative disease / factor and becomes able to maintain normal spontaneous respiration through the upper airway. When this is achieved it is rendered as successful decannulation and if patient cannot maintain normal respiration through upper airway after removing the tracheostomy tube out even if causative disease / factor persists no more is considered as failed decannulation.

Weaning: It is shifting of the patient from assisted mechanical ventilation to normal spontaneous respiration. It is measured in terms of early or late weaning.

Timing of Tracheostomy: It is the time / day when the orotracheal intubation is replaced by the tracheostomy tube insertion after doing tracheostomy. It is counted from the day one when the patient is placed on mechanical ventilation through orotracheal intubation.
All the patients during the mentioned duration irrespective of any age and sex were included in the study. There Physicians were requsted to do tracheostomy in ICU to prolong mechanical ventilation. Tracheostomy was performed by orotracheal tube which was replaced by tracheostomy tube through which the mechanical ventilation continued. All those patients who got emergency tracheostomy first and later on after few hours or days shifted to mechanical ventilation because of the failure of maintaining effective oxygen saturation through spontaneous respiration were excluded from the study as our study focused on the timing of the elective tracheostomy and all those patients who expired despite having elective tracheostomy and assisted mechanical ventilation in ICU were also excluded from the study. This was because the assessment of parent disease mortality was not the purpose of this study. As to assess the outcome of tracheostomy in terms of decannulation and weaning in mechanically ventilated patients was one of the prime objectives and not the mortality because the mortality is generally not due to the tracheostomy and its timing but due to the severity of initial causative disease.

The medical records of all those patients meeting the above mentioned inclusion and exclusion criteria were collected from the intensive care unit of the hospital for required information. Age, Sex, initial medical or surgical disease, timing of elective tracheostomy after orotracheal intubation, place of elective tracheostomy, time of weaning from mechanical ventilation and patterns were noted on a separate Proforma.

Frequencies were used to express the descriptive variables, SPSS version 20 was used to analyze data.

\section{RESULTS:}

During the previous two years of study total 551 patients underwent tracheostomy. Out of these, 42(7.6\%) patients got elective tracheostomy on the request of attending physician of ICU for the purpose of prolonged mechanical ventilation. From these 42 patients were initially on endotrachealintubation for mechanical ventilation which was later on replaced by tracheostomy tube after tracheostomy. Elective tracheostomy of all these patients was performed in operation theatre. Regarding the indications of this procedure; it was found that 20 out of 42 (47.6\%) patients were having Guillain Barre Syndrome(GBS), $07(16.6 \%)$ patients were of encephalitis and in $06(14.2 \%)$ patients was indicated due to tetanus. Cerebrovascular accident (CVA) was found in 02(4.7\%) patients, Traumatic brain injury (TBI) was present in $02(4.7 \%)$ patients and 02(4.7\%) patients were found with Para Phenylene Diamine (PPD / Black stone) poisoning. Myasthenia gravis, acid ingestion and HELLP syndrome were present in one patient equally $(2.3 \%)$. (Table 1$)$. Regarding age distribution $26(61.9 \%)$ patients were adults and 16(38\%) were children (38\%). Mean age was 22 years. GBS was the most common indication among the two age group of adult 11(42.3\%) and 
Relationship of Timings and Outcome of Tracheostomy Among Patients Requiring Prolonged Mechanical Ventilation

children $9(56.2 \%$ ) respectively-(Table 1$)$. In this study; 24 $(57.1 \%)$ were male patients and $18(42.8 \%)$ females. Again GBS was the most common indication among both genders(Table 2).

The time when the elective tracheostomy was performed on these 42 patients was also recorded and mainly this was dependent on the request of attending physician of ICU. The time when elective tracheostomy was performed ranged between 7-10 days among 40 patients except 02 patients (4.7\%) where the request for the procedure was sent beyond 02 weeks, one on $20^{\text {th }}$ day and second on $25^{\text {th }}$ day of mechanical ventilation with orotracheal intubation and the mean was $9^{\text {th }}$ day. All those patients (40) who were operated and shifted to tracheostomy tube from orotracheal tube between 7-10 days were weaned off from mechanical ventilation earlier (within one month) after ICU management $(95.2 \%)$ and likewise were successfully decannulated at the time of discharge. 02 patients $(4.7 \%)$ who were operated for elective tracheostomy on $20^{\text {th }} \& 25^{\text {th }}$ day both had to face difficulty in early weaning and failed decannulation of tracheostomy tube $(100 \%)$ and hence their hospital stay was prolonged. Out of these 02 patients one patient was of head trauma (TBI) and one was of CVA (50\%) (Graph 1). There was no drop out in the results.

Table 1: Elective Tracheostomy in Adults and Children with Indications

\begin{tabular}{|c|c|c|c|}
\hline Indications & $\begin{array}{c}\text { Adults (26 } \\
\text { Patients) } \\
(69.9 \%)\end{array}$ & $\begin{array}{c}\text { Children (16 } \\
\text { Patients) } \\
(38 \%)\end{array}$ & $\mathrm{N}=42$ \\
\hline GBS & $11(42.3 \%)$ & $9(56.2 \%)$ & $20(47.6 \%)$ \\
\hline M.Encephalitis & $05(19.2 \%)$ & $02(12.5 \%)$ & $07(16.6 \%)$ \\
\hline Tetanus & $03(11.5 \%)$ & $03(18.7 \%)$ & $06(14.2 \%)$ \\
\hline CVA & $02(7.6 \%)$ & - & $02(4.7 \%)$ \\
\hline PPD & $02(7.6 \%)$ & - & $02(4.7 \%)$ \\
\hline TBI & $01(3.8 \%)$ & $01(6.25 \%)$ & $02(4.7 \%)$ \\
\hline M.Gravis & $01(3.8 \%)$ & - & $01(2.3 \%)$ \\
\hline HELLP Syndrome & $01(3.8 \%)$ & - & $01(2.3 \%)$ \\
\hline Acid Ingestion & - & $01(6.25 \%)$ & $01(2.3 \%)$ \\
\hline
\end{tabular}

Table 2: Indications of Elective Tracheostomy among Males and Females

\begin{tabular}{|c|c|c|c|}
\hline Indications & $\begin{array}{c}\text { Males (24) } \\
\text { Patients) } \\
(57.1 \%)\end{array}$ & $\begin{array}{c}\text { Females (18 } \\
\text { Patients) } \\
(42.8 \%)\end{array}$ & $\mathrm{N}=42$ \\
\hline GBS & $13(54.1 \%)$ & $07(38.8 \%)$ & $20(47.6 \%)$ \\
\hline M.Encephalitis & $04(16.6 \%)$ & $03(16.6 \%)$ & $07(16.6 \%)$ \\
\hline Tetanus & $03(12.5 \%)$ & $03(16.6 \%)$ & $06(14.2 \%)$ \\
\hline CVA & $02(8.3 \%)$ & - & $02(4.7 \%)$ \\
\hline TBI & $02(8.3 \%)$ & - & $02(4.7 \%)$ \\
\hline PPD & - & $02(11.1 \%)$ & $02(4.7 \%)$ \\
\hline M.Gravis & - & $01(5.5 \%)$ & $01(2.3 \%)$ \\
\hline Acid Ingestion & - & $01(5.5 \%)$ & $01(2.3 \%)$ \\
\hline HELLP Syndrome & - & $01(5.5 \%)$ & $01(2.3 \%)$ \\
\hline
\end{tabular}

Graph 1: Relation of pattern of weaning and mode of Decannulation of TT with the day when elective tracheostomy done in the patients having traumatic brain injury (TBI) and cerebrovascular accident (CVA).

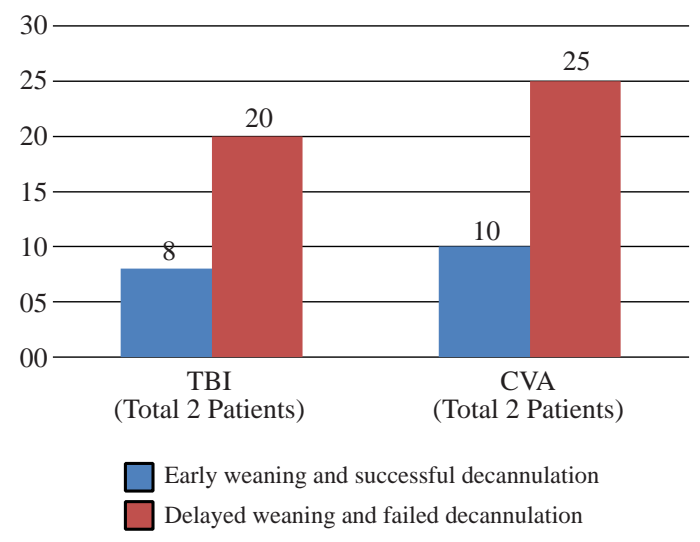

\section{DISCUSSION:}

The study focused on important clinical pathological indications of prolonged mechanical ventilation with tracheostomy for the patients coming to the settings of our hospital. In this study the neuromuscular paralysis turns out to be the commonest cause leading to prolonged mechanical ventilation and tracheostomy. There is paucity of work done on finding out the incidence and prevalence of different causative diseases for which patients undergo tracheostomy to continue prolonged mechanical ventilation. Generally due to heavy traumatic causalities coming to tertiary care hospitals, head injuries with coma are thought to be the leading indications for prolonged mechanical ventilation but in this study we observed neuroparalytic causes to remain on the top of the list during past two years and revealed Gullain Barre Syndrome (GBS) as the commonest medical condition responsible (47.6\%) whereas the head trauma / Traumatic Brain Injury ( TBI) was seen in 2 patients $(4.7 \%)$, and turned out to be the only non surgical traumatic cause for putting the patients on prolonged mechanical ventilation with elective tracheotomy. Padhi and colleagues in their study revealed $50 \%$ of the patients requiring elective tracheostomy for prolonged ventilation were of traumatic brain injury(TBI $)^{2}$. Datta et.al. in their study of 50 tracheostomies during two years found $2 \%$ cases of $\mathrm{GBS}^{3}$. Francois and Christian in their retrospective trial on critically ill patients of ICU found that the most frequent indication of tracheostomy was forthe prolonged mechanical ventilation ${ }^{12}$.

In our study $57 \%$ were male patients \& $43 \%$ were female patients. Datta et.al. in their study described the gender distribution as $80 \%$ of males \& $20 \%$ of females ${ }^{3}$. Almost every patient requiring prolonged mechanical ventilation needs replacement of orotracheal intubation with tracheostomy tube. Boubaker et.al. in their retrospective comparative analysis found that replacing tracheostomy tube in ICU patients for prolonged ventilation carried 
benefits of decreased ventilator induced lung complication but unchanged hospital stay and mortality ${ }^{13}$. In another cohort study conducted by Christophe and colleagues published in 2007 revealed no added benefit of elective tracheostomy among ICU patients in terms of disease mortality ${ }^{4}$.

In our study on 42 ICU patients requiring tracheostomy for prolonged ventilation, there were 26 adult patients $(62 \%)$ and 16 were children (38\%). Some studies have been conducted exclusively on children and infants who underwent tracheostomies. In Agha Khan University Hospital Karachi Pakistan, Prem Kumar et.al. did a retrospective study on the children requiring elective tracheostomy for mechanical ventilation and mentioned that during the 5 years of observational study $2.2 \%$ of all children admitted in ICU needed elective tracheostomy with male predominance $(60 \%)^{11}$. Whereas in our study out of total 16 children who underwent tracheostomy, 14 were male (87.5\%). Considerable work has been done in the world on the timing of tracheostomy in patients on prolonged mechanical ventilation. The timing is counted from the day one of the orotracheal intubation and mechanical ventilation. In our study; 95.2\% of the patients had tracheotomy between $7-10^{\text {th }}$ day with mean of $9^{\text {th }}$ day. These patients had successful early weaning from mechanical ventilation and decannulation of tracheostomy. $4.7 \%$ of the patients who were operated late ( $20^{\text {th }} \& 25^{\text {th }}$ day) for tracheostomy had to suffer delayed weaning and failed decannulation of tracheostomy tubeat the time of discharge from ICU. Failed decannulation was due to subglottic stenosis. Researchers have argued the benefits of early tracheostomy in mechanically ventilated patients and mentioned the depending factors like nature of disease and selection of patients.Charles has mentioned that the decision of early tracheostomy in mechanically ventilated patients should be individualized, however traumatized patients especially with head injury / TBI can be benefited more from early tracheostomy ${ }^{5}$. He also advocated that the tracheostomy should be within 7 days of intubation 5 . Julian and colleagues in their randomized pilot trial have found that early tracheostomy in stroke related patients remained feasible and $\mathrm{safe}^{8}$. Van Der et.al. in a retrospective subgroup analysis found early weaning of 31 neurological / neurosurgical patients from ventilator with early tracheostomy as compared to the other subgroup ${ }^{14}$.

Schauer and colleagues in their non randomized trial mentioned early tracheostomy with greater benefit in terms of duration of mechanical ventilation and hospital stay ${ }^{15}$. Aissaoui et al in a retrospective comparative analysis have found significantly better outcome in a group of patients who had tracheostomy before day 7 as compared to the other group where patients had tracheostomy after day $7^{16}$.

A retrospective study of 531 mechanically ventilated patients by Arabi and colleagues found increased duration of mechanical ventilation and hospital stay associated with the timing of tracheostomy ${ }^{17}$. A meta analysis of 6 randomized controlled trials on 406 adult patients in 2005 found significant reduction of mechanical ventilation with early tracheostomy (mean 8.5 days $)^{18}$. On the contrary Barquist et al in their prospective trials on traumatized patients did not find significant difference in the outcome of mechanically ventilated patients who had tracheostomy on day 8 as compared to those who had tracheostomy on $28^{\text {th }}$ day ${ }^{19}$. Heidler and colleagues in their prospective multicentric study from 2014 to 2016 on 831 neurologically ill tracheostomized weaned patients found significantly negatively associated predictors with increasing age for early decannulation $^{20}$. A study including case reports by Mitaka found facilitated weaning from prolonged mechanical ventilation if high flow oxygen was delivered through tracheostomy showing the enhanced benefit of doing tracheostomy in such patients requiring prolonged mechanical ventilation ${ }^{21}$. Likewise regarding the timing of tracheostomy Khammas and Dawood in their comparative analytical study described the clinical outcomes of early and late tracheostomy in the patients on prolonged mechanical ventilation. They found notable benefits like early weaning, lesser sedation and low risk of ventilation associated pneumonia in the patients who had early tracheostomy (within 1-10 days) than in those who had late tracheostomy (11-21 days) ${ }^{22}$. Yasir-ud-Din Hoti and colleagues studied early versus late tracheostomy in the patients of severe head injury and found early tracheostomy more beneficial in terms of ICU stay and mortality ${ }^{23}$. This study was carried out and published in Pakistan. Another local study conducted by Fazal and colleagues in 2018 assessing the secondary brain injury in the patients of severe head injury having early / late tracheostomy found increased morbidity and mortality in group of patients having late tracheostomy ${ }^{24}$. Amir Sabih Hydri et.al. studied the patterns of weaning and decannulation in the patients of ICU having surgical tracheostomy and interestingly found no prognostic dependence upon timing of tracheostomy but rather on underlying causative disease $\mathrm{e}^{25}$.

The limitations of our study were the small sample size, lack of detailed assessment on tracheostomy induced and mechanical ventilation associated complications in ICU patients due to its retrospective nature of the study design. It is recommended that large sample size study will be conducted to assess such complications like stomal injuries, tracheal ring injuries, pneumothorax, stomal stenosis, vascular erosions, nerve trauma, bleeding, infection and mechanical ventilation associated Pneumonia. Furthermore the preferred technique of doing tracheostomy and the place / venue of procedure are also the points of debate and question. Percutaneous dilatation tracheostomy on bed side in Intensive care unit has been advocated in some centers of the world. ${ }^{25}$

Hence wherever done and by any method adopted, tracheostomy should be performed as early as possible in the patients requiring prolonged mechanical ventilation. 


\section{CONCLUSION:}

Different medical and surgical causes / indications were present in the patients requiring tracheostomy for prolonged mechanical ventilation. Neuroparalytic diseases more frequent in patients, with increasing trends among male adult patients.

\section{REFERENCES:}

1. Joao A, CaioA, Vicente F, Martin C, Nikei T, Luiz E. Tracheostomy in the ICU:Is it worthwhile? J Bras Penumol 2007; 33(6):687-90.

2. PadhiR,Panda BN,Jagati S. Effects of tracheostomy on respiratory mechanics in critically ill patients requiring prolonged mechanical ventilation.Indian Journal Of Research And Reports In Medical Sciences2013Jan;Vol.3(1):1-4.

3. DattaRK,VishwanathB,PuneetPJ,MerinB , Kumari TL.Tracheostomy: Our Experince.Research in Otolaryngology 2015; 4(2):29-33.

4. Christophe C, Corinne A,Francois V, Maite G, Arnaud L, Dany $\mathrm{T}$ et al. Tracheostomy does not improve the outcome of patients requiring prolonged mechanical ventilation:A Propensity analysis. Crit Care Med 2007; 35(1): 132-37.

5. Charles G Durbin.Tracheostomy:Why, When and How ? Respir Care 2010;55(8): 1056-68.

6. Sanabria A, Gomez X, Vega V, Dominguez L, Osorio C. Prediction of prolonged mechanical ventilation for intensive care unit patients: A cohort study. Colomb Med 2013; 44:18488 .

7. Charles GD,FaarcJM, MichaelPP, LisaKM. Should Tracheostomy Be Performed as Early as 72 Hours in Patients Requiring Prolonged Mechanical Ventilation? Respir Care 2010; 55 (1): 76-83.

8. Julian B,PetraS, Yvonne H, Michaila A, Jan Oliver N, Sven P. Stroke-Related Early Tracheostomy Versus Prolonged OrotrachealIntubation in Neurocritical Care Trial.Stroke 2013;44: 21-28.

9. Alison E, MeixiaL,StephenC, Michael R, Shreve R, Mynard M. et al.Tracheostomy for Infants Requiring Prolonged Mechanical Ventilation:10 Years Experience. Pediatrics 2013 Apr;131:e1491-e496.

10. SidmanJD,JaguanA,CouserRJ.Tracheostomy and decannulation rates in a level 3 neonatal intensive care unit: a 12 year study. Laryngoscope2006;116(1):136-39.

11. Prem KM,Muhammad RK,Anwarul H. Elective Tracheostomy in Mechanically Ventilated Children. Journal of the College of Physicians and Surgeons of Pakistan2012; 22(6) : 414-15.

12. Francois B,ChristianM.Indications, Timing and Techniques of Tracheostomy in 152 French ICUs. CHEST 2005; 127 : 1347-352.
13. Boubaker C, Abdelhamid H, Abdellatif B, Said M. Tracheostomy versus prolonged intubation in medical intensive care unit patients. SIGNA VITAE2009; 4 (1) : 21-23.

14. Vander Lely AJ, Veelo DP, Dongelmans DA, Korevaar J, Vroom M, Schultz M. Time to wean after tracheostomy differs among subgroups of critically ill patients: retrospective analysis in a mixed medical / surgical intensive care unit. Respir Care 2006; 51: 1408-415.

15. Schauer JM, Engle LL, Maugher DT, Cherry RA. Does acuity matter? Optimal timing of tracheostomy stratified by injury severity . J Trauma 2009; 66 (1): 220-25.

16. Aissaoui Y, Azendour H, Balkhi H, Haimeur C, Kamili D, AtmaniM.. Timing of Tracheostomy and outcome. Ann FrAnesth et de Reanim 2007;26(6) : 496-501.

17. Arabi YM, Alhashemi JA, Tamim HM, Esteban A, Haddad SH, Dawood A et al.The impact of time to tracheostomy on mechanical ventilation duration, length of stay, and mortality in intensive care unit patients. J Crit Care 2009; 24(3): 43540.

18. Griffiths J, Barber VS, Morgan L, Young JD. Systematic review and meta-analysis of studies of the timing of tracheostomy in adult patients undergoing artificial ventilation. BMJ 2005; 330 (7502) : 1243.

19. Barquist ES, AmorteguiJ, HallalA, Giannotti G, Whinney R, Alzamel $\mathrm{H}$ et al. Tracheostomy in ventilator dependent trauma patients; a prospective, randomized intention- to- treat study.J Trauma 2006; 60(1): 91-97.

20. Heidler MD, Salzwedel A, Jobges M , Luck O, Dohle C, Seifert M et al. Decannulation of tracheostomized patientsafter long-term mechanical ventilation, results of a prospective multicentric study in German neurological early rehabilitation hospitals. BMC Anesthesiol 2018; 18(1): 65.

21. Mitaka C, Odoh M, Saloh D, Hashiouchi T, Inada E. Highflow oxygen via tracheostomy facilitates weaning from prolonged mechanical ventilation in patients with restrictive pulmonary dysfunction: two case reports. J Med Case Rep 2018; 12(1): 292.

22. Khammas AH, Dawood MR. Timing of Tracheostomy in Intensive Care Unit Patients. Int Arch Otorhinolaryngol 2018; 22(4): 437-42.

23. Yasir DH, Irfan M, Aziz A, Aamir H, Ishaque K. Early Versus Late Tracheostomy in patients with Severe Head Injury. Pak J Neuro Surg 2012;16(2):66-74.

24. Fazal G, Muhammad S, Muhammad I. Secondary Brain Injury in Early and Late Tracheostomy in Severe Head Injury patients. Pak J Neuro Surg 2018;22(2):71-5.

25. Amir SH, Irfan S, Fatima S, Munazza JS, Junaid H, Quratul- Ain R. Outcome of surgical tracheostomy in an intensive care unit. Rawal Med J 2017;42(1):116-20. 